\title{
Qualidade de vida de pacientes estomizados atendidos no Centro de Atenção à Saúde
}

\section{de Sergipe}

Quality of life evaluation in ostomized patients attended at Centro de Atenção à Saúde de Sergipe

Evaluación de la calidad de vida en pacientes estomáticos atendidos en el Centro de Atenção à

Saúde de Sergipe

Recebido: 21/09/2021 | Revisado: 30/09/2021 | Aceito: 04/10/2021 | Publicado: 05/10/2021

Adrielle Almeida de Jesus

ORCID: https://orcid.org/0000-0003-0072-5506

Universidade Tiradentes, Brasil

E-mail: adrielle.a.j@gmail.com

Maria Luiza Coelho de Sousa

ORCID: https://orcid.org/0000-0002-3820-9729

Universidade Tiradentes, Brasil

E-mail: luizamariacst@ outlook.com

Jéssica Abreu Silva

ORCID: https://orcid.org/0000-0001-7287-6165

Universidade Tiradentes, Aracaju, Brasil

E-mail: abreu.jessiica3@gmail.com

Maria Clara do Nascimento Gomes

ORCID: https://orcid.org/0000-0002-5191-6575

Universidade Tiradentes, Brasil

E-mail: mariaclaramcg@hotmail.com

Mariana Alma Rocha de Andrade

ORCID: https://orcid.org/0000-0002-3516-6997

Universidade Tiradentes, Brasil

E-mail: nana.alma@gmail.com

Manuela Naiane Lima Barreto

ORCID: https://orcid.org/0000-0002-4487-9227

Universidade Tiradentes, Brasil

E-mail: manuela.naiane@gmail.com

Victoria Cavalcanti de Souza

ORCID: https://orcid.org/0000-0002-2622-856X

Universidade Federal do Paraná, Brasil

E-mail: vc.vi10@hotmail.com

Liliane Almeida Santos

ORCID: https://orcid.org/0000-0003-0731-8700

Universidade Tiradentes, Brasil

E-mail: liliane.alsan@gmail.com

Arthur Valido Deda

ORCID: https://orcid.org/0000-0003-0627-4769 Universidade Federal de Sergipe, Brasil

E-mail: arthurdeda@live.com

Vivian Fernandes dos Santos

ORCID: https://orcid.org/0000-0002-8926-1244

Universidade Federal de Sergipe, Brasil

E-mail: vivianfernandess@hotmail.com.br

Renata Lima Batalha de Andrade

ORCID: https://orcid.org/0000-0002-7531-2311

Universidade Tiradentes, Brasil

E-mail: renatinhalba0@gmail.com

Maria Julia Nardelli

ORCID: https://orcid.org/0000-0002-5203-2069

Universidade Tiradentes, Brasil

E-mail: juvetnardelli@yahoo.com.br

Carla Viviane Freitas de Jesus

ORCID: https://orcid.org/0000-0002-7775-6610

Universidade Tiradentes, Brasil

E-mail: carlavfj@gmail.com

Sônia Oliveira Lima

ORCID: https://orcid.org/0000-0002-3257-2412

Universidade Tiradentes, Brasil

E-mail: sonialima.cirurgia@gmail.com 


\title{
Resumo
}

Objetivo: Avaliar perfil epidemiológico, qualidade de vida (QV) de pacientes com estomia intestinal. Metodologia: Estudo transversal e quantitativo, de janeiro à maio de 2021, com estomizados maiores de 18 anos, atendidos no Centro de Atenção à Saúde de Sergipe. Aplicou-se questionário sociodemográfico, dados sobre a estomia e avaliação da QV (Stoma-QoL - variante brasileira), sobre os domínios físico, psicológico, ambiental, relações sociais e sexuais. Utilizou-se testes Shapiro-Wilk e Mann- Whitney $(\mathrm{p} \leq 0,05)$. Resultados: Avaliou-se 100 pacientes, 54\% masculino, $70 \%$ maior de 50 anos, casados, cor parda, renda média de 2573,00 reais, da $1^{\text {a }}$ à $4^{\text {a }}$ série do ensino fundamental. Destes $93 \%$ eram colostomizados e $53 \%$ de caráter definitivo, a principal causa foi câncer colorretal. Identificou-se que $58 \%$ não se sentem sexualmente atraentes e $45 \%$ se sentem ansiosos quando a bolsa enche. Os pacientes que afirmaram não sentir sintomas negativos após a estomia têm melhor QV em relação ao demais que sentiram os sintomas, 20\% relatam acompanhamento psicológico e desses 75\% tiveram melhora na QV. Ter apoio familiar e de amigos são bons preditores para melhor QV. Conclusão: Não houve diferença entre os sexos dos estomizados, a maioria maior de 50 anos, casados, com baixa renda e escolaridade. Predominou a colostomia de caráter definitivo pelo câncer colorretal. No geral, apresentaram boa QV, condizendo com sua autopercepção, com insatisfação na sexualidade, e questões mecânicas do estoma. O atendimento especializado, acompanhamento psicológico e participação em grupos com a mesma condição, melhora o enfrentamento e auxilia no conhecimento dos cuidados, reduzindo os impactos negativos causados na QV.

Palavras-chave: Estomia; Saúde; Qualidade de vida; Bolsa de colostomia.

\begin{abstract}
Objective: Evaluate epidemiological profile and quality of life (QF) of patients with intestinal ostomy. Methodology: Transversal and quantitative research, from january to may 2021, compound by ostomized patients older than 50 years attended at the Centro de Atenção à Saúde de Sergipe in Aracaju/SE. A sociodemographic questionnaire was applied, including ostomy data and life quality evaluation tool (Stoma-QoL-Brazilian variant), about physical, psychological, enviromental, social and sexual relationships domains. The Shapiro-Wilk and Mann-Whitney test was used ( $\mathrm{p} \leq 0.05)$. Results: 100 patients were evaluated, 54 males, $70 \%$ older than 50 years, married, brown skin, avareage income $\mathrm{R} \$ 2573,00$ and schooling between 1 st to 4 th grade. $93 \%$ were colostomized and $53 \%$ in permanent condition, mostly because of colorectal cancer treatment. It was identified that $58 \%$ do not see themselves as sexually attractive and $45 \%$ get anxious about bag filling. Patients who denied feeling negative symptons after stoma have better QF in comparison to who felt, $20 \%$ reported psychological support and $75 \%$ of those have had improvement in their QF. Having family and friends support are good predictors related to better QF. Conclusion: It was not observed difference between the gender, most of them were married, with low income and low schooling. Definitive colostomy by colorectal cancer predominated. In general, patients presented good life quality, agreeing with their selfperspective, despite of sexual dissatisfaction and mechanical issues regarding the colostomy bag. Specialized treatment, psychological support and participation in groups improve the coping related to this condition and contributes with knowledge about self-caring, reducing negative impacts in QF.
\end{abstract}

Keywords: Ostomy; Health; Quality of life; Colostomy bag.

\section{Resumen}

Objetivo: Evaluar perfil epidemiológico, calidad de vida (CV) de pacientes con ostomía intestinal. Metodología: Estudio transversal y cuantitativo, de enero a mayo de 2021, con pacientes ostomizados mayores de 18 años, atendidos en el Centro Sanitario de Sergipe. Se aplicó un cuestionario sociodemográfico, datos de ostomía y evaluación de la CV (Stoma -QoL - variante brasileña), en los dominios físico, psicológico, ambiental, social y de relaciones sexuales. Se utilizaron las pruebas de Shapiro-Wilk y Mann-Whitney $(\mathrm{p} \leq 0.05)$. Resultados: Se evaluaron 100 pacientes, $54 \%$ hombres, $70 \%$ mayores de 50 años, casados, de color castaño, ingreso promedio de 2573,00 reales, desde el $1^{\circ}$ al $4^{\circ}$ grado de la escuela primaria. De estos el $93 \%$ fueron colostomías y el $53 \%$ fueron definitivas, la principal causa fue el cáncer colorrectal. Se identificó que el 58\% no se siente sexualmente atractivo y el $45 \%$ se siente ansioso cuando la bolsa está llena. Los pacientes que dijeron no sentir síntomas negativos después de la ostomía tuvieron una mejor calidad de vida en relación a los otros que sintieron los síntomas, el $20 \%$ refirió seguimiento psicológico y de estos el $75 \%$ tuvo una mejoría en su calidad de vida. Tener el apoyo de familiares y amigos es un buen indicador de una mejor calidad de vida. Conclusión: No hubo diferencia entre los sexos de los pacientes ostomizados, la mayoría casados, con bajos ingresos y educación. Predominó la colostomía definitiva por cáncer colorrectal. En general, tuvieron una buena calidad de vida, consistente con su autopercepción, insatisfacción con la sexualidad y problemas mecánicos del estoma. La atención especializada, el seguimiento psicológico y la participación en grupos con la misma condición mejora el afrontamiento y ayuda en el conocimiento del cuidado, reduciendo los impactos negativos que se generan en la $\mathrm{CV}$.

Palabras clave: Ostomia; Salud; Calidad de vida; Bolsa de colostomía. 


\section{Introdução}

A estomia é uma medida cirúrgica, realizada para auxiliar a função de determinado órgão. Feita através de um desvio no trajeto habitual, que vai do interior da cavidade conectando-a ao ambiente exterior (Santos et al., 2016). É um procedimento indicado quando a restauração da continuidade intestinal é contra-indicada ou não é imediatamente viável devido ao estado clínico do paciente (Dulk et al., 2009; Wong et al., 2013).

O trato gastrointestinal é o principal local para realização de estomias, podem ser colostomias ou ileostomias, dependendo do lugar onde são criadas (intestino delgado ou grosso, respectivamente) e são feitas principalmente para que haja eliminação intestinal (Luz et al., 2014; Salomé \& Almeida, 2014). As estomias podem ser temporárias ou definitivas, sendo necessárias devido à doenças colorretais com características malignas, ou benignas (trauma, doenças inflamatórias intestinais, anormalidades congênitas, obstrução de cólon, isquemias) (Doughty, 2005). Sendo o câncer colorretal a principal causa de estomização, principalmente em idosos (Mols et al., 2014).

Os estomizados enfrentam diversas alterações desde físicas, psicológicas, sociais, até sexuais. Ocorre mudança da autoestima, que passa a interferir na qualidade de vida, relacionada à saúde. O modo usual de eliminação das fezes é alterado e com isso os pacientes passam a vivenciar uma nova perspectiva de vida, que se associa à existência do estoma e uso da bolsa coletora (Torres et al., 2016; Nascimento et al., 2011; Sales et al., 2010). A estomização faz com que o paciente necessite criar outras formas de conviver com as diversas mudanças dessa transição psicossocial. Sentimentos como medo, angústia, tristeza e desamparo são relatados ao uso da bolsa coletora, por permitir vivências autodepreciativas, além de autoeficácia reduzida, senso de incapacitação crônica, inutilidade, e outras questões emocionais (Batista et al., 2011).

Uma estomia têm efeitos generalizados na vida cotidiana, que se combinam para reduzir o funcionamento geral e a qualidade de vida. Vão desde usar uma bolsa de ostomia por baixo da roupa, possibilidade de irritações cutâneas repetidas e outras complicações, vazamentos e necessidade de mudar o sistema de bolsa com muita frequência, dificuldades com a seleção da dieta e controle do intestino. (Kimura et al., 2013; Faria et al., 2018; Sousa et al., 2012).

Na vida desses pacientes, questões como trabalho, lazer, sexualidade passam por diversas alterações e muitos têm seus sentimentos de insegurança e temor de rejeição acentuados (Batista et al., 2011). O medo de ocorrer "acidentes" com a bolsa de estomia, como odores desagradáveis, eliminação de gases, ou rompimento dela, faz com que as relações sociais se dificultem, limitando a interação dos pacientes estomizados com outras pessoas, e suas vivências fora de casa (Dos santos et al., 2016). A forma como se enfrenta essa situação, e a intensidade de como essa condição afeta a vida do indivíduo, depende de cada um e da sua capacidade de adaptação física e emocional. Ainda assim, pode existir sentimentos de exclusão, constrangimento e rejeição, afetando diretamente numa qualidade de vida menor, como consequência da estomização (Salome, Almeida \& Silveira et al., 2014).

A inserção da bolsa coletora requer desde o pré-operatório uma preparação dos profissionais de saúde envolvidos no andamento desses pacientes, para que as alterações físicas e emocionais, consequentes da cirurgia, sejam consideradas. É importante valorizar a reabilitação e inserção eficiente do estomizado, com ênfase no autocuidado (Sampaio et al., 2008; Mendonça et al., 2007). Preparar o paciente antes da criação planejada da ostomia inclui a seleção do local do estoma, suporte emocional e educação do estomizado, bem como planejamento antecipado para alta subsequente, cuidados de reabilitação contínua para o paciente e seus entes (Ferreira et al., 2017).

Nesse contexto se insere a necessidade de se investigar a qualidade de vida desses pacientes. Esse conceito vem englobar a saúde física, o estado psicológico, o nível de independência, as relações sociais, as crenças pessoais e a relação com aspectos significativos do meio ambiente (Borges et al., 2007; Aguiar et al., 2019). O presente estudo se justifica pelo fato de que aspectos relacionados à qualidade de vida desse grupo de pacientes, como grau de dependência, capacidade de realização de atividades cotidianas, laborais, autoestima, dentre outras, podem afetar diretamente a percepção de saúde e qualidade de 
vida. Assim, o objetivo deste estudo foi avaliar a qualidade de vida de pacientes com estomia intestinal, determinando o perfil sociodemográfico, bem como os aspectos relacionados aos domínios: físico, psicológico, ambiental, de relações sociais e sexuais, que compõem a qualidade de vida.

\section{Metodologia}

Trata-se de um estudo transversal, com abordagem quantitativa. A pesquisa foi realizada entre os meses de janeiro à maio de 2021, por meio de questionários aplicados em pacientes estomizados intestinais, SUS dependentes, que buscam os serviços ofertados no Centro de Atenção à Saúde de Sergipe. O tamanho da amostra atendeu aos critérios de inclusão e exclusão, como critérios de inclusão foram usados pacientes estomizados acima de 18 anos, de ambos os sexos e que demonstraram interesse através da assinatura do Termo de Consentimento Livre e Esclarecido (TCLE). Foram excluídos do estudo pacientes que apresentaram distúrbios cognitivos que prejudicasse a interpretação do questionário.

No ambulatório de estomia, no período que foi realizada a pesquisa, havia 135 pacientes que atenderam aos critérios de inclusão e exclusão, calculou-se a amostra representativa pela fórmula de Pocock e, encontrou-se a quantidade mínima de 100 pacientes, não havendo perdas ao longo do estudo. Coletaram-se os dados com 100 pacientes, estimando-se uma prevalência esperada de 50\%, margem de erro de 5\%, e intervalo de confiança de $95 \%$.

Foram utilizados dois instrumentos, sendo o primeiro um questionário estruturado para avaliar questões sociodemográficas, com o objetivo de conhecer o perfil dos pacientes participantes do estudo, além de dados sobre sua estomia. O questionário confeccionado pela equipe continha perguntas como nome, idade, naturalidade, procedência, sexo, renda familiar, escolaridade e dados sobre estomia, como o tipo, temporalidade da bolsa, motivo e tempo de uso da estomia, tempo da troca de bolsa, quem e quantas vezes a bolsa é higienizada, além de perguntas referentes ao acompanhamento psicológico após a estomia, melhora da qualidade de vida depois desse acompanhamento e com que frequência o paciente possuia sentimentos negativos.

O segundo instrumento utilizado foi o Questionário STOMA -QoL - Validado para o português variante brasileira, "Qualidade de vida relacionada à saúde e perfil nutricional de portadores de derivação intestinal - colostomia e ileostomia" (Oliveira, 2017). O questionário possui as seguintes variáveis: Fico ansioso quando a bolsa está cheia?, Fico preocupado de a bolsa soltar ?, Sinto a necessidade de saber onde fica o banheiro mais próximo?, Fico preocupado que a bolsa possa cheirar mal?, Fico preocupado com os barulhos que o estoma faz?, Preciso descansar durante o dia ?, A bolsa limita a escolhas de roupas que eu possa usar?, Sinto-me cansado durante o dia? O estoma não me faz sentir sexualmente atraente?, Durmo mal durante a noite? Preocupa- me que a bolsa faça barulho?, Eu me sinto envergonhado com o meu corpo por causa do meu

estoma?, É difícil para mim passar uma noite fora de casa?, É difícil esconder o fato de que eu uso uma bolsa?, Preocupa- me que a minha condição seja um problema para as pessoas que me são próximas?, Eu evito contato físico mais próximo com os meus amigos?, O estoma torna difícil para mim estar com outras pessoas?, Eu tenho medo de conhecer novas pessoas?, Eu me sinto sozinho, mesmo quando estou com outras pessoas?, Preocupa- me que a minha família se sinta desconfortável perto de mim?. O questionário Stoma-QoL é composto por 20 perguntas. Avaliadas em uma escala de Likert de quatro pontos (nunca = 4 , raramente $=3$, às vezes $=2 \mathrm{e}$ sempre $=1$ ). A soma do respostas dá uma pontuação total variando de $20 \mathrm{a} 80$ e foi convertido para uma escala de 0 a 100, em que uma pontuação alta representa um nível mais alta a qualidade de vida, enquanto pontuações mais baixas representam baixos níveis de qualidade de vida (Pietro, Thorsen \& Juul, 2005).

Após aplicação dos questionários os dados foram compilados no programa Microsoft Office Excel versão 2016. Foram obtidas variáveis qualitativas e quantitativas, onde a análise dos dados foi realizada de duas formas, descritiva e inferencial. Para as variáveis qualitativas a análise descritiva procedeu com a categorização dos dados e obtenção das 
respectivas frequências e percentuais, e o cálculo da média, mediana, desvio, padrão, intervalo interquartil, mínimo e máximo das variáveis quantitativas.

Inicialmente foi verificada a normalidade das distribuições de variáveis quantitativas através do teste de Shapiro-Wilk como não foi observado normalidade (p-valor $<0,05$ ), adotou-se testes não paramétricos para a análise (Shapiro \& Wilk, 1965). Para o cruzamento entre as variáveis quantitativas e qualitativas, utilizou-se o Kruskal-Wallis para comparações múltiplas e o teste de Dunn para determinar quais desses grupos são diferentes, e foram calculadas as medianas e o intervalo interquartil para cada grupo (Kruskal \& Wallis, 1952; Dunn, 1954). Para as variáveis com duas categorias, o teste escolhido foi o de Mann-Whitney (Mann \& Whitney, 1947). As diferenças entre as medianas foram representadas por letras (a, b e c), as que compartilham a mesma letra indica a igualdade entre as medianas, já as que possuem letras diferentes indicam a diferença entre as medianas.

Em todos os testes de hipótese realizados a conclusão foi obtida através da interpretação do p-valor. Adotando um nível de significância de 5\%, sempre que o p-valor calculado for menor que 0,05 diremos que há associação entre as variáveis analisadas. O software utilizado foi o R, versão 4.0.4 (Team, 2021).

Antes da aplicação do questionário, o paciente lia e assinava o Termo de Consentimento Livre e esclarecido, que deixava clara a garantia de sigilo sobre identidade do participante. A pesquisa foi aprovada pelo Comitê de Ética em Pesquisa da Universidade Tiradentes, com CAEE: 37321420.0.0000.5371 e parecer $\mathrm{n}^{\circ} 4.464 .869$.

\section{Resultados}

Nesse estudo foram entrevistados 100 indivíduos estomizados, sendo 54\% do sexo masculino e $46 \%$ do feminino. Desses, $70 \%$ tinham 50 anos ou mais de idade, 50\% eram casados e $46 \%$ pardas. Em relação ao grau de escolaridade, $21 \%$ afirmaram ter estudado da $1^{\mathrm{a}}$ à $4^{\mathrm{a}}$ série do ensino fundamental. A renda familiar variou de 0 a 40 mil reais, com média de $\mathrm{R} \$$ 2.573 ( \pm 5.414$)$ (Tabela 1$)$. 
Tabela 1. Características sociodemográficas dos pacientes estomizados avaliados no Centro de Atenção à Saúde de Sergipe, no período de janeiro à maio de 2021.

\begin{tabular}{|c|c|c|c|c|}
\hline Variável & Média & Desvio-Padrão & Mínimo & Máximo \\
\hline RENDA FAMILIAR & 2.573 & 5.414 & 0 & 40.000 \\
\hline Variável/Categoria & & $\mathbf{N}$ & & $\%$ \\
\hline \multicolumn{5}{|l|}{ SEXO } \\
\hline Feminino & & 46 & & 46 \\
\hline Masculino & & 54 & & 54 \\
\hline \multicolumn{5}{|l|}{ IDADE } \\
\hline 18 a 25 anos & & 3 & & 3 \\
\hline 26 a 33 anos & & 8 & & 8 \\
\hline 34 a 41 anos & & 9 & & 9 \\
\hline 42 a 49 anos & & 10 & & 10 \\
\hline 50 anos ou mais & & 70 & & 70 \\
\hline \multicolumn{5}{|l|}{ ESTADO CIVIL } \\
\hline Solteiro & & 29 & & 29 \\
\hline Casado(a) / mora com um(a) companheiro(a) & & 50 & & 50 \\
\hline Separado(a) / divorciado(a) / desquitado(a) & & 14 & & 14 \\
\hline Viúvo(a) & & 7 & & 7 \\
\hline \multicolumn{5}{|l|}{ COR } \\
\hline Branco(a) & & 22 & & 22 \\
\hline Pardo(a) & & 46 & & 46 \\
\hline Negro(a) & & 29 & & 29 \\
\hline Amarelo(a) & & 2 & & 2 \\
\hline Indígena & & 1 & & 1 \\
\hline \multicolumn{5}{|l|}{ ESCOLARIDADE } \\
\hline Não estudou & & 14 & & 14 \\
\hline Da $1^{\mathrm{a}}$ à $4^{\mathrm{a}}$ série do ensino fundamental (antigo primário) & & 21 & & 21 \\
\hline Da $5^{\mathrm{a}}$ à $8^{\mathrm{a}}$ série do ensino fundamental (antigo ginásio) & & 18 & & 18 \\
\hline Ensino médio (antigo $2^{\circ}$ grau) incompleto & & 11 & & 11 \\
\hline Ensino médio completo & & 19 & & 19 \\
\hline Ensino superior incompleto & & 7 & & 7 \\
\hline Ensino superior completo & & 6 & & 6 \\
\hline Pós-graduação & & 4 & & 4 \\
\hline
\end{tabular}

Fonte: Autores.

Do total de entrevistados, 93\% possuíam colostomias e 7\% tinham ileostomia. Quando perguntado o motivo do uso, $56 \%$ afirmaram ser devido a câncer colorretal e $80 \%$ afirmaram ter estomia a mais de um ano. Em 53\% o uso da bolsa tinha caráter definitivo e 50\% realizam a troca no intervalo de 3 à 7 dias. Em relação à quantidade de vezes do esvaziamento da bolsa, $42 \%$ afirmaram esvaziar mais de 3 vezes por dia, seguido por 38\% de 2 a 3 vezes. Quando perguntando quem higieniza a bolsa, $84 \%$ pessoas responderam que elas mesmas fazem a limpeza. Em relação ao acompanhamento psicológico após a estomia 20\% afirmaram terem sido acompanhados, destes, $75 \%$ disseram que houve melhora na qualidade de vida. Depois da estomia, 30\% afirmaram que nunca sentiram sintomas negativos, $24 \%$ algumas vezes e $23 \%$ raramente e sempre (Tabela 2 ). 
Tabela 2. Variáveis relacionadas às características clínicas, cuidados e qualidade de vida dos pacientes estomizados avaliados no Centro de Atenção à Saúde de Sergipe, no período de janeiro à maio de 2021.

\begin{tabular}{|c|c|c|}
\hline Variável/Categoria & $\mathbf{N}$ & $\%$ \\
\hline \multicolumn{3}{|l|}{ TIPO DA ESTOMIA } \\
\hline Colostomia & 93 & 93 \\
\hline Ileostomia & 7 & 7 \\
\hline \multicolumn{3}{|l|}{ MOTIVO DO USO } \\
\hline Câncer colorretal & 56 & 56 \\
\hline Diverticulite & 6 & 6 \\
\hline Doenças inflamatórias (Doença de Chron/ Retocolite) & 1 & 1 \\
\hline Outro & 37 & 37 \\
\hline \multicolumn{3}{|l|}{ A QUANTO TEMPO ESTÁ COM A ESTOMIA } \\
\hline Menos de 1 mês & 4 & 4 \\
\hline De 6 a 1 ano & 16 & 16 \\
\hline mais de 1 ano & 80 & 80 \\
\hline \multicolumn{3}{|l|}{ TEMPORALIDADE DA BOLSA } \\
\hline Definitiva & 53 & 53 \\
\hline Provisória & 47 & 47 \\
\hline \multicolumn{3}{|l|}{ DE QUANTO EM QUANTO TEMPO TROCA A BOLSA } \\
\hline De 1 a 3 dias & 44 & 44 \\
\hline De 3 a 7 dias & 50 & 50 \\
\hline Mais de 7 dias & 6 & 6 \\
\hline \multicolumn{3}{|l|}{ QUANTAS VEZES/DIA ESVAZIA A BOLSA } \\
\hline $1 \mathrm{vez}$ & 20 & 20 \\
\hline 2 a 3 vezes & 38 & 38 \\
\hline Mais de 3 vezes & 42 & 42 \\
\hline \multicolumn{3}{|l|}{ QUEM HIGIENIZA SUA BOLSA } \\
\hline Eu mesmo & 84 & 84 \\
\hline Outra pessoa & 16 & 16 \\
\hline \multicolumn{3}{|c|}{ FEZ ACOMPANHAMENTO PSICOLOGICO APÓS A ESTOMIA } \\
\hline Sim & 20 & 20 \\
\hline Não & 80 & 80 \\
\hline \multicolumn{3}{|c|}{ SE A RESPOSTA ANTERIOR FOR SIM, SUA QUALIDADE DE VIDA MELHOROU? } \\
\hline Sim & 15 & 75 \\
\hline Não & 5 & 25 \\
\hline \multicolumn{3}{|c|}{ DEPOIS DA ESTOMIA SENTIU SINTOMAS NEGATIVOS? } \\
\hline Sempre & 23 & 23 \\
\hline Algumas vezes & 24 & 24 \\
\hline Raramente & 23 & 23 \\
\hline Nunca & 30 & 30 \\
\hline
\end{tabular}

Fonte: Autores.

Verificou-se que $35 \%$ dos entrevistados ficavam ansiosos sempre que a bolsa estava cheia, $45 \%$ sempre se preocupavam se a bolsa iria soltar, $35 \%$ não via necessidade em saber onde fica o banheiro mais próximo ao sair, $50 \%$ não se preocupavam se a bolsa de estomia iria cheirar mal, 37\% se limitavam ao escolher as roupas, 58\% não se sentiam sexualmente atraentes, $51 \%$ mencionavam não dormir mal durante a noite, $44 \%$ não sentiram vergonha do corpo, $48 \%$ não tinham dificuldade em passar a noite fora de casa, $48 \%$ não escondiam o fato de usar a bolsa de estomia, $57 \%$ não se preocupavam em a estomia ser um problema para as pessoas próximas, $67 \%$ referiram nunca ter dificuldade em estar com outras pessoas, $71 \%$ nunca tinham medo de conhecer pessoas novas, $65 \%$ nunca se sentiam sozinhas, $81 \%$ relatavam não sentir preocupação em a família sentir desconforto em estar por perto (Tabela 3). 
Tabela 3. Apresenta a distribuição da frequência das respostas do questionário Stoma-QoL, aplicados em indivíduos estomizados atendidos no Centro de Atenção à Saúde de Sergipe, no período de janeiro a maio de 2021.

\begin{tabular}{|c|c|c|}
\hline Variável/categoria & $\mathbf{N}$ & $\%$ \\
\hline \multicolumn{3}{|c|}{ FICA ANSIOSO QUANDO A BOLSA ESTÁ CHEIA? } \\
\hline Sempre & 35 & 35 \\
\hline Algumas vezes & 17 & 17 \\
\hline Raramente & 18 & 18 \\
\hline Nunca & 30 & 30 \\
\hline \multicolumn{3}{|c|}{ FICA PREOCUPADA SE A BOLSA SE SOLTAR } \\
\hline Sempre & 45 & 45 \\
\hline Algumas vezes & 12 & 12 \\
\hline Raramente & 15 & 15 \\
\hline Nunca & 28 & 28 \\
\hline \multirow{2}{*}{\multicolumn{3}{|c|}{$\begin{array}{l}\text { SINTO A NECESSIDADE DE SABER ONDE FICA O BANHEIRO MAIS } \\
\text { PRÓXIMO }\end{array}$}} \\
\hline & & \\
\hline Sempre & 35 & 35 \\
\hline Algumas vezes & 17 & 17 \\
\hline Raramente & 9 & 9 \\
\hline Nunca & 39 & 39 \\
\hline \multicolumn{3}{|c|}{ FICO PREOCUPADO QUE A BOLSA POSSA CHEIRAR MAL } \\
\hline Sempre & 31 & 31 \\
\hline Algumas vezes & 11 & 11 \\
\hline Raramente & 8 & 8 \\
\hline Nunca & 50 & 50 \\
\hline \multicolumn{3}{|c|}{ FICO PREOCUPADA COM OS BARULHOS QUE O ESTOMA FAZ } \\
\hline Sempre & 38 & 38 \\
\hline Algumas vezes & 14 & 14 \\
\hline Raramente & 15 & 15 \\
\hline Nunca & 33 & 33 \\
\hline \multicolumn{3}{|c|}{ PRECISO DESCANSAR DURANTE O DIA } \\
\hline Sempre & 16 & 16 \\
\hline Algumas vezes & 16 & 16 \\
\hline Raramente & 10 & 10 \\
\hline Nunca & 58 & 58 \\
\hline \multicolumn{3}{|c|}{ A BOLSA LIMITA A ESCOLHAS DE ROUPAS QUE EU POSSA USAR } \\
\hline Sempre & 37 & 37 \\
\hline Algumas vezes & 18 & 18 \\
\hline Raramente & 9 & 9 \\
\hline Nunca & 36 & 36 \\
\hline \multicolumn{3}{|c|}{ SINTO-ME CANSADO DURANTE O DIA } \\
\hline Sempre & 14 & 14 \\
\hline Algumas vezes & 15 & 15 \\
\hline Raramente & 14 & 14 \\
\hline Nunca & 57 & 57 \\
\hline \multicolumn{3}{|c|}{ O ESTOMA NÃO ME FAZ SENTIR SEXUALMENTE ATRAENTE } \\
\hline Sempre & 58 & 58 \\
\hline Algumas vezes & 15 & 15 \\
\hline Raramente & 8 & 8 \\
\hline Nunca & 19 & 19 \\
\hline \multicolumn{3}{|c|}{ DURMO MAL DURANTE A NOITE } \\
\hline Sempre & 16 & 16 \\
\hline Algumas vezes & 17 & 17 \\
\hline Raramente & 16 & 16 \\
\hline Nunca & 51 & 51 \\
\hline \multicolumn{3}{|c|}{ PREOCUPA-ME QUE A BOLSA FAÇA BARULHO } \\
\hline Sempre & 48 & 48 \\
\hline Algumas vezes & 12 & 12 \\
\hline Raramente & 9 & 9 \\
\hline Nunca & 31 & 31 \\
\hline
\end{tabular}




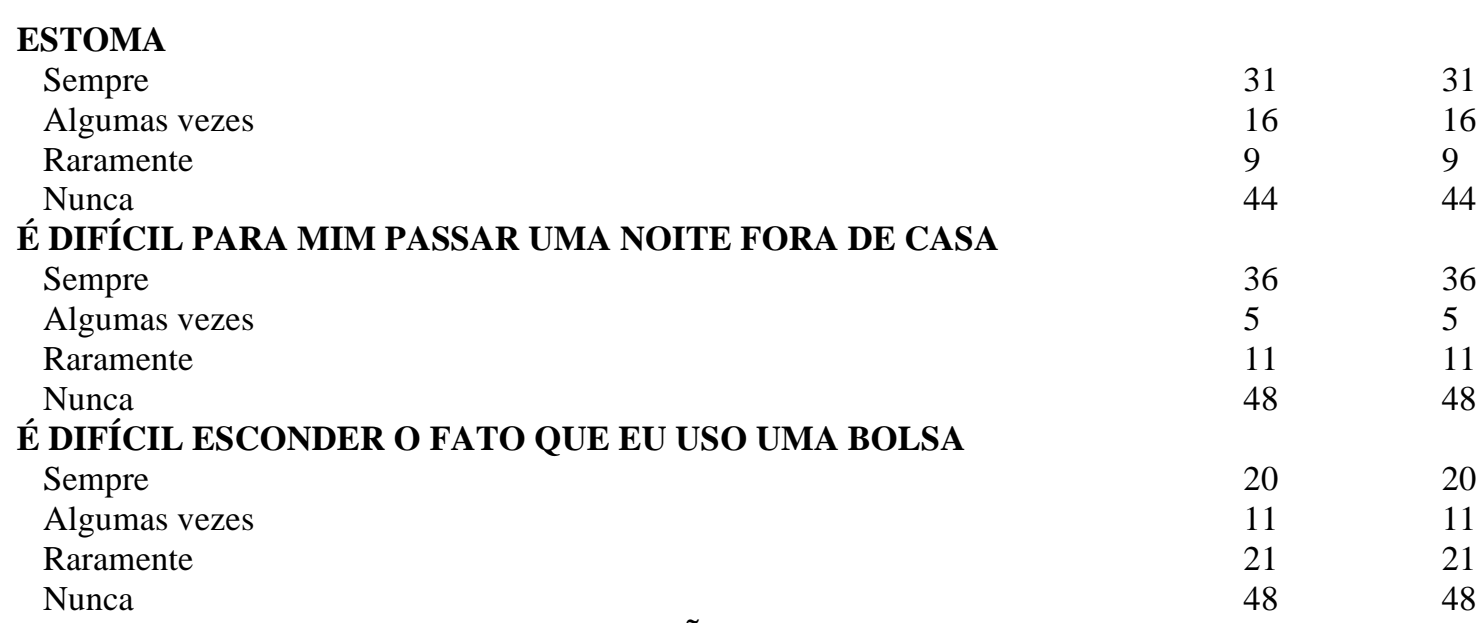

PREOCUPA-ME QUE A MINHA CONDIÇÃO SEJA UM PROBLEMA PARA AS PESSOAS QUE ME SÃO PRÓXIMAS

$\begin{array}{lcc}\text { Sempre } & 12 & 12 \\ \text { Algumas vezes } & 11 & 11 \\ \text { Raramente } & 20 & 20 \\ \text { Nunca } & 57 & 57\end{array}$

EU EVITO CONTATO FÍSICO MAIS PRÓXIMO COM OS MEUS AMIGOS

Sempre

Algumas vezes $\quad 5 \quad 5$

$\begin{array}{llll}\text { Raramente } & 14 & 14\end{array}$

Nunca

O ESTOMA TORNA DIFÍCIL PARA MIM ESTAR COM OUTRAS

PESSOAS

Sempre $\quad 17 \quad 17$

Algumas vezes $\quad 13$

Raramente $\quad 3 \quad 3$

$\begin{array}{llll}\text { Nunca } & 67 & 67\end{array}$

EU TENHO MEDO DE CONHECER NOVAS PESSOAS

$\begin{array}{llll}\text { Sempre } & 14 & 14\end{array}$

$\begin{array}{lll}\text { Algumas vezes } & 10 & 10\end{array}$

Raramente $\quad \begin{array}{ll}10 & 5\end{array}$

$\begin{array}{lll}\text { Nunca } & 71 & 71\end{array}$

EU ME SINTO SOZINHO, MESMO QUANDO ESTOU COM OUTRAS

PESSOAS

$\begin{array}{lll}\text { Sempre } & 9 & 9\end{array}$

$\begin{array}{lll}\text { Algumas vezes } & 17 & 17\end{array}$

$\begin{array}{lll}\text { Raramente } & 9 & 9\end{array}$

$\begin{array}{lll}\text { Nunca } & 65 & 65\end{array}$

PREOCUPA-ME QUE A MINHA FAMÍLIA SE SINTA

DESCONFORTÁVEL PERTO DE MIM

Sempre

Algumas vezes

Raramente 6

Nunca

Fonte: Autores.

Na relação entre as características socioeconômicas e o score total não houve diferença estatisticamente significativa entre a qualidade de vida e o sexo, idade, estado civil, escolaridade ( $\mathrm{p}>0,05)$ (Tabela 4).

Tabela 4. Associação entre as características socioeconômicas e o score total, de pacientes estomizados atendidos no Centro de atenção à saúde de Sergipe no período de janeiro a maio de 2021. 


\begin{tabular}{|c|c|c|c|}
\hline Variável/Categoria & Mediana & Intervalo Interquartil & P-valor \\
\hline \multicolumn{4}{|l|}{ SEXO } \\
\hline Feminino & 60,0 & 36,7 & \multirow[t]{2}{*}{0,06725} \\
\hline Masculino & 70,8 & 32,5 & \\
\hline \multicolumn{4}{|l|}{ IDADE } \\
\hline 18 a 25 anos & 70,0 & 10,0 & \multirow[t]{5}{*}{0,35119} \\
\hline 26 a 33 anos & 64,2 & 47,1 & \\
\hline 34 a 41 anos & 55,0 & 21,7 & \\
\hline 42 a 49 anos & 46,7 & 41,3 & \\
\hline 50 anos ou mais & 69,2 & 34,6 & \\
\hline \multicolumn{4}{|l|}{ ESTADO CIVIL } \\
\hline Solteiro & 60,0 & 25,0 & \multirow[t]{4}{*}{0,58749} \\
\hline Casado(a) / mora com um(a) companheiro(a) & 70,0 & 30,0 & \\
\hline Separado(a) / divorciado(a) / desquitado(a) & 50,8 & 35,0 & \\
\hline Viúvo(a) & 65,0 & 53,3 & \\
\hline \multicolumn{4}{|l|}{ ESCOLARIDADE } \\
\hline Não estudou & 60,8 & 32,1 & \multirow[t]{8}{*}{0,11418} \\
\hline Da $1^{\mathrm{a}}$ à $4^{\mathrm{a}}$ série do ensino fundamental (antigo primário) & 51,7 & 31,7 & \\
\hline Da $5^{\mathrm{a}}$ à $8^{\mathrm{a}}$ série do ensino fundamental (antigo ginásio) & 61,6 & 20,4 & \\
\hline Ensino médio (antigo $2^{\circ} \mathrm{grau}$ ) incompleto & 70,0 & 35,0 & \\
\hline Ensino médio completo & 76,7 & 23,3 & \\
\hline Ensino superior incompleto & 75,0 & 25,8 & \\
\hline Ensino superior completo & 60,8 & 59,6 & \\
\hline Pós-graduação & 50,8 & 12,5 & \\
\hline
\end{tabular}

Fonte: Autores.

Houve diferença estatisticamente significativa entre a qualidade de vida e se o paciente sentiu sintomas negativos após a estomia $(\mathrm{p}<0,05)$. Observou-se que os pacientes que afirmaram não sentir sintomas negativo após a estomia têm maior qualidade de vida em relação ao demais que sentiram os sintomas (Tabela 5). 
Tabela 5. Associação entre as características clínicas e o score total de pacientes estomizados atendidos no Centro de Atenção à Saúde de Sergipe no período de janeiro a maio de 2021.

\begin{tabular}{|c|c|c|c|}
\hline Variável/Categoria & Mediana & $\begin{array}{l}\text { Intervalo } \\
\text { Interquartil }\end{array}$ & P-valor \\
\hline \multicolumn{4}{|l|}{ TIPO DA ESTOMIA } \\
\hline Colostomia & 68,3 & 33,3 & \multirow[t]{2}{*}{0,40970} \\
\hline Ileostomia & 56,7 & 23,3 & \\
\hline \multicolumn{4}{|l|}{ MOTIVO DO USO } \\
\hline Câncer colorretal & 61,7 & 37,5 & \multirow[t]{3}{*}{0,18255} \\
\hline Diverticulite & 77,5 & 19,6 & \\
\hline Outro & 68,3 & 26,7 & \\
\hline \multicolumn{4}{|c|}{ A QUANTO TEMPO ESTÁ COM A ESTOMIA } \\
\hline Menos de 1 mês & 72,5 & 7,5 & \multirow[t]{3}{*}{0,84914} \\
\hline De 6 a 1 ano & 71,7 & 34,2 & \\
\hline Mais de 1 ano & 65,8 & 33,3 & \\
\hline \multicolumn{4}{|c|}{ TEMPORALIDADE DA BOLSA } \\
\hline Definitiva & 68,3 & 36,7 & \multirow[t]{2}{*}{0,67342} \\
\hline Provisória & 66,7 & 29,2 & \\
\hline \multicolumn{4}{|c|}{ DE QUANTO EM QUANTO TEMPO TROCA A BOLSA } \\
\hline De 1 a 3 dias & 64,2 & 32,5 & \multirow[t]{3}{*}{0,65809} \\
\hline De 3 a 7 dias & 67,5 & 32,9 & \\
\hline Mais de 7 dias & 73,3 & 14,2 & \\
\hline \multicolumn{4}{|c|}{ QUANTAS VEZES/DIA ESVAZIA A BOLSA } \\
\hline $1 \mathrm{vez}$ & 68,3 & 21,7 & \multirow[t]{3}{*}{0,08417} \\
\hline 2 a 3 vezes & 71,7 & 32,5 & \\
\hline Mais de 3 vezes & 51,7 & 40,8 & \\
\hline \multicolumn{4}{|c|}{ QUEM HIGIENIZA SUA BOLSA } \\
\hline Eu mesmo & 67,5 & 32,1 & \multirow[t]{2}{*}{0,81409} \\
\hline Outra pessoa & 65,8 & 34,2 & \\
\hline \multicolumn{4}{|c|}{ FEZ ACOMPANHAMENTO PSICOLOGICO APÓS A ESTOMIA } \\
\hline Sim & 61,7 & 32,5 & \multirow[t]{2}{*}{0,95875} \\
\hline Não & 68,3 & 32,1 & \\
\hline \multicolumn{4}{|c|}{ SE A RESPOSTA ANTERIOR FOR SIM, SUA QUALIDADE DE VIDA } \\
\hline \multicolumn{4}{|c|}{ MELHOROU? } \\
\hline Sim & 60,0 & 37,5 & \multirow[t]{2}{*}{0,48433} \\
\hline Não & 61,7 & 21,7 & \\
\hline \multicolumn{4}{|c|}{ DEPOIS DA ESTOMIA SENTIU SINTOMAS NEGATIVOS? } \\
\hline Sempre & $38,3^{\mathrm{a}}$ & 39,2 & \multirow[t]{4}{*}{0,00000} \\
\hline Algumas vezes & $57,5^{\mathrm{ab}}$ & 25,8 & \\
\hline Raramente & $68,3^{\mathrm{bc}}$ & 28,3 & \\
\hline Nunca & $87,5^{\mathrm{c}}$ & 19,2 & \\
\hline
\end{tabular}

Fonte: Autores.

\section{Discussão}

No presente estudo verificou-se que a maioria dos pacientes com estomia intestinal tinham idade maior que 50 anos, concordando com o estudo de Kimura et al. (2013), no qual a idade média dos pacientes foi de 56 anos. Quanto ao sexo, houve concordância com os dados encontrados na literatura, que mostram que a maioria dos estomizados é do sexo masculino (Salomé \& Almeida, 2014). A maior causa encontrada foram as neoplasias malignas colorretais, em consonância com o estudo de (Torres et al., 2015). O que denota a importância de uma investigação clínica adequada em pacientes com sintomas sugestivos de câncer colorretal, para que se consiga um diagnóstico precoce, evitando assim a necessidade de realizar a estomia.

Em relação ao estado civil dos pacientes, a maioria eram casados, concordando com a literatura (Sousa et al., 2012). De acordo com a raça a maioria era parda, apresentando discrepância com dados encontrados na literatura, na qual mostram 
que a maioria dos estomizados são brancos (Borges et al., 2007). O grau de escolaridade como em outras literaturas Aguiar et al. (2019); Ferreira et al. (2017), permeou o baixo nível de escolaridade, no qual $21 \%$, afirma ter cursado da $1^{\text {a a }} 4^{\mathrm{a}}$ série do ensino fundamental, o que pode ser um fator para não prevenção do câncer colorretal, principal causa de estomização, devido à escassa formação recebida sobre os fatores que causam esta doença, incluindo hábitos alimentares (Silva et al., 2010).

As colostomias sobressaíram em detrimento das ileostomias, contudo, Landmann et al. (2019), cita que ambas devem se adaptar a uma alteração significativa no corpo e na imagem, bem como desafios relacionados ao gerenciamento de odores e gases, atividade sexual, viagens e limitações alimentares. A maioria dos pacientes são estomizados há mais de 1 ano, concordando a literatura pertinente Faria et al. (2018), e prevalecendo no presente estudo as estomias de caráter definitivo. O que parece interferir numa melhor qualidade de vida desses indivíduos em detrimento das temporárias, uma vez que, a estomia temporária faz com que os pacientes demorem de reajustar suas vidas, já que estes esperam voltar a sua condição anterior (Souza et al., 2011).

No questionário para avaliação da qualidade de vida, a principal queixa dos estomizados foi que sempre ficam ansiosos quando a bolsa enche, enquanto aproximadamente a metade afirmou que sempre ficam com medo da bolsa se soltar e se preocupam com o barulho que o estoma faz, concordando com a literatura pertinente (Silva et al., 2010). É notório que as limitações, constrangimento, medo de acidentes provocados pela bolsa, e a fisiologia intestinal, interferem no status social. Pontos importantes que prejudicam ou até impedem o retorno desses pacientes às atividades diárias e aos momentos de lazer, tendendo o paciente portador de bolsa coletora a passar mais tempo dentro de casa (Silva et al., 2016; Batista et al.,2011).

No presente estudo, embora a maioria significativa dos pacientes com sintomas negativos tenha obtido uma baixa pontuação na qualidade de vida, observou-se que, na análise geral os estomizados obtiveram uma boa avaliação, incluindo uma boa percepção da sua imagem corporal e do seu convívio social, além da adaptação favorável ao uso da bolsa coletora. A maior parte dos domínios analisados, obtiveram escores indicando boa qualidade de vida. Resultados como esses, se assemelham aos estudos como o de Faria et al. (2018); Wong et al. (2013), que identificaram boa qualidade de vida em pacientes estomizados com os mesmos aspectos sócio-demoragráficos. É importante o conhecimento dos profissionais de saúde sobre as necessidades inerentes aos cuidados dos estomas, com o intuito de melhorar a assistência e com isso permitir uma melhor qualidade de vida dos estomizados.

Em relação a sintomas físicos e demais itens, como fadiga e sono após a estomização e uso da bolsa coletora, houve um bom score, mostrando a não alteração desses domínios nos pacientes. Achados que diferem de estudos como os de Ferreira et al. (2017); Torres et al. (2015), os quais mencionam nos resultados, insônia e cansaço como principais sintomas físicos dos colostomizados. Mais da metade dos pacientes mencionam que sempre se sentem sexualmente menos atraentes, semelhantes a pesquisas que mostraram prejuízo no âmbito sexual (Paula, Takahashi, \& Paula, 2009; Kiliç, Taycan \& Belli, 2007; Souza et al., 2011). Aspectos esses, que denotam a necessidade de reconstituição do trânsito intestinal, logo que possível, reduzindo os constrangimentos sofridos no âmbito sexual desses pacientes.

Nas respostas envolvendo o relacionamento social de estomizados, com amigos próximos e familiares, quase 2/3 dos pacientes nunca se preocuparam em se sentir desconfortável perto de familiares, assim como, mais da metade nunca se sente sozinho e nunca se preocupam em sua nova condição ser um problema para pessoas próximas. Visto isso, como contribuição positiva nos resultados da análise geral, que demonstra boa qualidade de vida dos estomizados. Confirma-se a importância do apoio familiar na adequação do ambiente e rotina à nova realidade do paciente com estomia, assim como citam Kimura et al. (2013), que a presença da família constitue um suprimento crucial para pacientes estomizados com câncer ajudando-os a lidar com os impactos emocionais e sociais. Portanto, verificou-se que a qualidade da vida está intimamente associada ao estado psicológico e as relações sociais desses pacientes. 
Em relação ao acompanhamento psicológico e ser participante da Associação Sergipana de Ostomizados e Amigos, inferiu-se que a maioria dos pacientes não fizeram acompanhamento e/ou não tiveram acesso a uma das duas alternativas, enquanto que os $20 \%$ que fizeram quase $2 / 3$ mencionaram melhora na qualidade de vida. O que concorda com as evidências científicas que relacionam melhor qualidade de vida em estomizados que possuem rede de apoio incluindo relações pessoais com familiares e amigos, relações com profissionais de saúde, para melhor seguimento pós estomia e relações sociais com outros pessoas estomizadas, com experiências de vida semelhantes (Cascais, Martini \& Almeida, 2007; Dabirian et al., 2011; Maruyama \& Zago, 2005).

\section{Conclusão}

O perfil sociodemográfico em pacientes colostomizados atendidos no CASE, não houve diferença entre os sexos, com média de idade maior que 50 anos, casados, de cor parda, que possuem uma renda média de 2573,00 reais e estudaram da $1^{\text {a à }}$ $4^{a}$ série do ensino fundamental. O principal motivo das estomias foi o câncer colorretal, sendo o tipo mais prevalente as colostomias, de caráter definitivo. A análise geral da qualidade de vida dos pacientes com estomia apresentou um bom escore, embora algumas facetas apresentadas mereçam atenção. Verificou-se que a estomia provoca uma redução da autoestima dos seus portadores, interferindo negativamente na atividade sexual, em questões físicas como barulhos intestinais, os quais os deixam ansiosos, assim como o enchimento e aderência da bolsa. A presença de sintomas negativos, apresentou correlação direta com má qualidade de vida.

É de suma importância que os estomizados recebam atendimento especializado, acompanhamento psicológico e participem de grupos com a mesma condição, a fim de que, esses pacientes enfrentem melhor esse período de tantas mudanças, incluindo imagem corporal, sexualidade e convívio social. O suporte adequado e continuado possibilita uma melhor compreensão dos cuidados referentes ao uso da bolsa, com intuito de reduzir os impactos negativos causados na qualidade de vida desses pacientes.

\section{Referências}

Aguiar, F. A. S., Pinheiro de Jesus, B., Cardoso Rocha, F., Barbosa Cruz, I., de Andrade Neto, G. R., Meira Rios, B. R., \& Batista Andrade, D. L. (2019). Colostomy And Self-Care: Meanings For Ostomized Patients. Journal of Nursing UFPE/Revista de Enfermagem UFPE, 13(1).

Batista, M. D. R. D. F. F., Rocha, F. C. V., Silva, D. M. G. D., \& Silva Júnior, F. J. G. D. (2011). Autoimagem de clientes com colostomia em relação à bolsa coletora. Revista Brasileira de enfermagem, 64, 1043-1047.

Borges, E. C., Camargo, G. C., Souza, M. O., Pontual, N. A., \& de Sá Novato, T. (2007). Qualidade de vida em pacientes ostomizados: uma comparação entre portadores de câncer colorretal e outras patologias* Quality of life in patients stomized: a comparison between carriers of colorectal neoplasms and other pathologies. Rev Inst Ciênc Saúde, 25(4), 357-63.

Cascais, A. F. M. V., Martini, J. G., \& Almeida, P. J. D. S. (2007). O impacto da ostomia no processo de viver humano. Texto \& Contexto-Enfermagem, 16, $163-167$.

Dabirian, A., Yaghmaei, F., Rassouli, M., \& Tafreshi, M. Z. (2011). Quality of life in ostomy patients: a qualitative study. Patient preference and adherence, 5,1 .

Dulk, M. D., Marijnen, C. A. M., Collette, L., Putter, H., Påhlman, L., Folkesson, J., \& Van De Velde, C. J. H. (2009). Multicentre analysis of oncological and survival outcomes following anastomotic leakage after rectal cancer surgery. Journal of British Surgery, 96(9), 1066-1075.

Doughty, D. (2005). Principles of ostomy management in the oncology patient. The Journal of Supportive Oncology, 3(1), 59-69.

Dunn, O. J. (1964). Multiple comparisons using rank sums. Technometrics, 6(3), 241-252.

Faria, F. L., Labre, M. M., de Sousa, I. F., \& de Almeida, R. J. (2018). Avaliação da qualidade de vida em pacientes com estomia intestinal. Arquivos de Ciências da Saúde, 25(2), 8-14.

Ferreira, E. D. C., Barbosa, M. H., Sonobe, H. M., \& Barichello, E. (2017). Self-esteem and health-related quality of life in ostomized patients. Revista brasileira de enfermagem, 70, 271-278.

Kiliç, E., Taycan, O., \& Bellı, A. K. (2007). The Effect of Permanent Ostomy on Body Image, Self-Esteem, Marital Adjustment, and Sexual Functioning. Turkish journal of psychiatry, 18(4). 
Kimura, C. A., Kamada, I., Guilhem, D., \& Monteiro, P. S. (2013). Quality of life analysis in ostomized colorectal cancer patients. Journal of Coloproctology $33,216-221$.

Kruskal, W. H., \& Wallis, W. A. (1952). Use of ranks in one-criterion variance analysis. Journal of the American statistical Association, 47(260), 583-621.

Landmann, R. G. (2019). Ileostomy or colostomy care and complications.

Luz, A. L. D. A., Luz, M. H. B. A., Antunes, A., Oliveira, G. S. D., Andrade, E. M. L. R., \& Miranda, S. M. (2014). Perfil de pacientes estomizados: revisão integrativa da literatura.

Mann, H. B., \& Whitney, D. R. (1947). On a test of whether one of two random variables is stochastically larger than the other. The annals of mathematical statistics, 50-60.

Maruyama, S. A. T., \& Zago, M. M. F. (2005). O processo de adoecer do portador de colostomia por câncer. Revista Latino-Americana de Enfermagem, 13, $216-222$.

Mendonça, R. S., Valadão, M., Castro, L., \& Camargo, T. C. (2007). A importância da consulta de enfermagem em pré-operatório de ostomias intestinais. Revista Brasileira de Cancerologia, 53(4), 431-435.

Mols, F., Lemmens, V., Bosscha, K., van den Broek, W., \& Thong, M. S. (2014). Living with the physical and mental consequences of an ostomy: a study among 1-10-year rectal cancer survivors from the population-based PROFILES registry. Psycho-Oncology, 23(9), 998-1004.

Nascimento, C. D. M. D. S., Trindade, G. L. B., Luz, M. H. B. A., \& Santiago, R. F. (2011). Vivência do paciente estomizado: uma contribuição para a assistência de enfermagem. Texto \& Contexto-Enfermagem, 20, 557-564.

Oliveira, A. L. (2017). Qualidade de vida relacionada à saúde e perfil nutricional de portadores de derivação intestinal-colostomia e íleostomia. Juiz de Fora (MG): Universidade Federal de Juiz de Fora.

Paula, M. A. B. D., Takahashi, R. F., \& Paula, P. R. D. (2009). Os significados da sexualidade para a pessoa com estoma intestinal definitivo. Revista Brasileira de Coloproctologia, 29(1), 77-82.

Prieto, L., Thorsen, H., \& Juul, K. (2005). Development and validation of a quality of life questionnaire for patients with colostomy or ileostomy. Health and Quality of Life Outcomes, 3(1), 1-10.

Rocha, F. C., da Silva, D. M., \& Júnior, F. J. (2011). Self-image of clients with colostomy related to the collecting bag. Revista brasileira de enfermagem, 64(6), 1043-1047.

Sales, C. A., Violin, M. R., Waidman, M. A. P., Marcon, S. S., \& Silva, M. A. P. D. (2010). Sentimiento de las personas ostomizadas: comprensión existencial. Revista da Escola de Enfermagem da USP, 44(1), 221-227.

Salomé, G. M., \& Almeida, S. A. D. (2014). Association of sociodemographic and clinical factors with the self-image and self-esteem of individuals with intestinal stoma. Journal of Coloproctology (Rio de Janeiro), 34, 159-166.

Sampaio, F. A. A., Aquino, P. D. S., Araújo, T. L. D., \& Galvão, M. T. G. (2008). Nursing care to an ostomy patient: application of the Orem s theory. Acta Paulista de Enfermagem, 21, 94-100.

Santos, O. J., Sauaia, E. N., Barros, A. K. D., Desterro, V. S., Silva, T., Venicius, M., \& Santos Sauaia, C. H. (2016). Children and adolescents ostomized in a reference hospital. Epidemiological profile. Journal of Coloproctology (Rio de Janeiro), 36, 75-79.

Shapiro, S. S., \& Wilk, M. B. (1965). An analysis of variance test for normality (complete samples). Biometrika, 52(3/4), 591-611.

Silva, E. S. D., Castro, D. S. D., Garcia, T. R., Romero, W. G., \& Primo, C. C. (2016). Tecnologia do cuidado à pessoa com colostomia: diagnósticos e intervenções de enfermagem. Revista Mineira de Enfermagem, 20.

Silva, D. G., Bezerra, A. L. Q., Siqueira, K. M., Paranaguá, T. T. D. B., \& Barbosa, M. A. (2010). Influência dos hábitos alimentares na reinserção social de um grupo de estomizados.

Siegel, S., \& Castellan Jr, N. J. (1975). Estatística não-paramétrica para ciências do comportamento. Artmed Editora.

Sousa, C. F., Brito, D. C., \& Branco, M. Z. P. C. (2012). Depois da colostomia... vivências das pessoas portadoras. Enfermagem em Foco, 3(1), 12-15.

Souza, P. C. M., da Costa, V. R. M., Maruyama, S. A. T., da Costa, A. L. R. C., Rodrigues, A. E. C., \& Navarro, J. P. (2011). As repercussões de viver com uma colostomia temporária nos corpos: individual, social e político. Revista Eletrônica de Enfermagem, 13(1), 50-9.

Tan, W. S., Tang, C. L., Shi, L., \& Eu, K. W. (2009). Meta-analysis of defunctioning stomas in low anterior resection for rectal cancer. Journal of British Surgery, 96(5), 462-472.

Team, R. C. (2013). R: A language and environment for statistical computing.

Torres, C. R. D., Andrade, E. M. L. R., Ribeiro, F. M. S., Gonçalves Neta, F. D. C. C., \& Luz, M. H. B. A. (2015). Qualidade de vida de pessoas estomizadas: revisão integrativa. Rev. enferm. UFPI, 117-122.

Wong, S. K., Young, P. Y., Widder, S., \& Khadaroo, R. G. (2013). A descriptive survey study on the effect of age on quality of life following stoma surgery. Ostomy Wound Manage, 59(12), 16-23. 